\title{
ON VALUATION RINGS THAT CONTAIN ZERO DIVISORS
}

\author{
JAMES A. HUCKABA ${ }^{1}$
}

\begin{abstract}
Let $R$ be a commutative ring with identity. A new proof is given of the theorem due to Samuel and Griffin which states that $R$ is integrally closed in its total quotient ring if and only if $R$ is the intersection of $B$-valuation rings. We then prove the main result of the paper: If $K$ is a $\pi$-regular ring, then $K$ admits only Prüfer rings as valuation rings.
\end{abstract}

1. A ring means a commutative ring with identity. If $(G,+)$ is a totally ordered abelian group, then a function $v$ from a ring $T$ into $G \cup\{\infty\}$ is an evaluation with value group $G$, if for all $x, y \in T$ :

(1) $v(x y)=v(x)+v(y)$

(2) $v(z+y) \geqq \min \{v(x), v(y)\}$;

(3) $v(1)=0$ and $v(0)=\infty$.

If $v$ maps $T$ onto $G \cup\{\infty\}$, then $v$ is called a valuation. For any value group $G$, let $G^{+}=\{\alpha \in G: \alpha \geqq 0\} \cup\{\infty\}$. Let $R$ be a subring of the ring $T$ and suppose that $v$ is an evaluation with value group $G$. We consider four conditions on $R$.

$\left(\mathrm{V}_{1}\right) R=v^{-1}\left(G^{+}\right)$.

$\left(\mathrm{V}_{2}\right) v$ is a valuation and $R=v^{-1}\left(G^{+}\right)$.

$\left(\mathrm{V}_{3}\right)$ If $P\left(X_{1}, \cdots, X_{r}\right)$ is a dominated polynomial over $R$, then $P\left(s_{1}, \cdots, s_{r}\right) \neq 0$ for every set $\left\{s_{i}\right\}_{i=1}^{r} \in T-R$. A dominated polynomial over $R$ is a polynomial of the form $P\left(X_{1}, \cdots, X_{r}\right)=X_{1}^{n(1)} \cdots X_{r}^{n(r)}+$ $\sum_{\delta} a_{\delta} X_{1}^{\delta(1)} \cdots X_{r}^{\delta(r)}$, with $a_{\delta} \in R$ and $(n(1), \cdots, n(r))>(\delta(1), \cdots, \delta(r))$ where the order is given by the ordered product of $r$ copies of the natural numbers.

$\left(\mathrm{V}_{4}\right) R$ contains a prime ideal $P$ such that if $B$ is a ring between $R$ and $T$, and if $Q$ is a prime ideal of $B$ contracting to $P$, then $R=B$.

If we assume that $T$ is a field, then the above definitions are equivalent and correspond to the classical definition of a valuation domain. In [13]

Received by the editors December 7, 1972.

AMS (MOS) subject classifications (1970). Primary 13A15, 13B20, 13 F05.

Key words and phrases. Commutative rings with zero divisors, valuation ring, Prüfer ring, von Neumann regular ring, integral closure of a ring.

${ }^{1}$ This research was partially supported by a University of Missouri Summer Research Grant. 
Samuel gives several generalizations of valuation theory to arbitrary commutative rings. It was this paper that introduced $\left(\mathrm{V}_{3}\right)$ and $\left(\mathrm{V}_{4}\right)$. Manis [10] proved the equivalence of $\left(\mathrm{V}_{2}\right)$ and $\left(\mathrm{V}_{4}\right)$, and at the present time it is commonly accepted that the best generalization of a valuation domain is a ring satisfying these conditions. Such rings are, therefore, called valuation rings. The concept that we call an evaluation was introduced by Bourbaki in [2] as his definition of a valuation. Hence, we call rings satisfying $\left(\mathrm{V}_{\mathbf{1}}\right)$ $B$-valuation rings.

Krull proved in 1932 [7] that the integral closure of an integral domain $D$ is the intersection of valuation domains between $D$ and its quotient field. Samuel generalized this in 1957 when he showed that the integral closure of an arbitrary commutative ring $R$ in an overring $T$ is the intersection of rings of type $\left(\mathrm{V}_{3}\right)$. Then in 1970 Griffin [5] proved the equivalence of $\left(\mathrm{V}_{1}\right)$ and $\left(\mathrm{V}_{3}\right)$, thus showing:

THEOREM 1. A ring $R$ is integrally closed in an overring $T$ if and only if $R$ is the intersection of $B$-valuation rings between $R$ and $T$.

The most important case of Theorem 1 is when $T$ is assumed to be the total quotient ring of $R$. For this situation we give, in $\S 2$, a new short proof of Theorem 1. Our method is completely different than that of Samuel and Griffin, and avoids the use of $\left(\mathrm{V}_{3}\right)$, the most complicated of the $\left(\mathrm{V}_{i}\right)$ axioms.

The methods of $\S 2$ are applicable to another important problem that arises when studying valuations on commutative rings-the problem of determining when a valuation ring is a Prüfer ring. The main part of this paper, $\S 3$, is devoted to this problem. We show that if $K$ is a $\pi$-regular ring, then every valuation subring of $K$ is Prüfer (Theorem 6).

2. Let $R$ be a ring, let $T(R)$ be its total quotient ring, and let $Q(R)$ denote its complete ring of quotients. For the definition and pertinent facts about the complete ring of quotients see Lambek's book [8]. In this section we give a short proof of Theorem 1 . We first need a result that is an easy generalization of one part of [6, Proposition 9]. The proof is similar to Griffin's proof and will not be repeated.

PROPOSITION 2. If $K$ is a von Neumann regular ring containing the ring $R$, then $R$ is integrally closed in $K$ if and only if $R$ is equal to an intersection of valuation rings of $K$ that contain $R$.

Note that in Proposition 2, $K$ is not necessarily the total quotient ring of $R$.

Proof of Theorem 1. We first assume that $R$ is a reduced ring (i.e., $R$ contains no nonzero nilpotent elements). Denote the integral closure of $R$ 
in $T(R)$ (resp., $Q(R)$ ) by $\bar{R}$ (resp., $\widetilde{R}$ ). By [8, p. 42], $Q(R)$ is a von Neumann regular ring. Proposition 2 implies that $\tilde{R}=\cap V_{\alpha}$, where the $V_{\alpha}$ are valuation rings of $Q(R)$. Thus, since $R \subseteq T(R) \subseteq Q(R)$,

$$
\bar{R}=\tilde{R} \cap T(R)=\left(\cap V_{\alpha}\right) \cap T(R)=\cap V_{\alpha}^{\prime},
$$

where $V_{\alpha}^{\prime}=V_{\alpha} \cap T(R)$. If $v_{\alpha}$ is the valuation on $Q(R)$ corresponding to $V_{\alpha}$, if $G_{\alpha}$ is the value group of $v_{\alpha}$, if $w_{\alpha}$ is the restriction of $v_{\alpha}$ to $T(R)$, and if $H_{\alpha}$ is the subgroup of $G_{\alpha}$ generated by the range of $w_{\alpha}$, then it is clear that $w_{\alpha}$ is an evaluation with value group $H_{\alpha}$ and that $w_{\alpha}^{-1}\left(H_{\alpha}^{+}\right)=V_{\alpha}^{\prime}$.

We now drop the assumption that $R$ is reduced. Let $N$ be the nilradical of $T(R)$, then $N_{0}=N \cap R$ is the nilradical of $R$. Canonically embed $R / N_{0}$ into $T(R) / N$ and embed $T(R) / N$ into $T\left(R / N_{0}\right)$ via the mapping $x / y+$ $N \rightarrow\left(x+N_{0}\right) /\left(y+N_{0}\right)$. Hence we may assume that

$$
R / N_{0} \subseteq T(R) / N \subseteq T\left(R / N_{0}\right) \subseteq Q\left(R / N_{0}\right) .
$$

It follows from the first paragraph of this proof that $\left(R / N_{0}\right)^{\sim}=\bigcap V_{\alpha}$, where each $V_{\alpha}$ is a valuation ring with respect to $Q\left(R / N_{0}\right)$. Again we assume that $v_{\alpha}$ and $G_{\alpha}$ are the valuation and valuation group associated with $V_{\alpha}$. Let $v_{\alpha}^{\prime}$ be the restriction of $v_{\alpha}$ to $T(R) / N$, and define $w_{\alpha}: T(R) \rightarrow$ $G_{\alpha} \cup\{\infty\}$ such that $w_{\alpha}(x)=v_{\alpha}^{\prime}(x+N)$. It follows that $w_{\alpha}$ is an evaluation on $T(R)$ such that $w_{\alpha}$ is infinite on $N$. If $H_{\alpha}$ is the subgroup of $G_{\alpha}$ generated by the range of $w_{\alpha}$, then $W_{\alpha}=w_{\alpha}^{-1}\left(H_{\alpha}^{+}\right)$is a $B$-valuation ring of $T(R)$.

It remains to show that $\bar{R}=\bigcap W_{\alpha}$. Each $W_{\alpha} \supseteq R$ and is integrally closed [13, Theorem 1], hence $\bar{R} \subseteq \bigcap W_{\alpha}$. Assume that $x \in \bigcap W_{\alpha}$. For each $\alpha$, $w_{\alpha}(x)=v_{\alpha}^{\prime}(x+N) \geqq 0$, which implies that $x+N$ is in the integral closure of $R / N_{0}$ in $Q\left(R / N_{0}\right)$. Write $(x+N)^{t}+\left(r_{1}+N_{0}\right)(x+N)^{t-1}+\cdots+\left(r_{t}+N_{0}\right)=$ $0+N$. We have $x^{t}+r_{1} x^{t-1}+\cdots+r_{t} \in N$, so there exists a positive integer $s$ such that $\left(x^{t}+r_{1} x^{t-1}+\cdots+r_{t}\right)^{s}=0$. This last equation shows that $x$ is integral over $R$. Therefore, $\bar{R}=\bigcap W_{\alpha}$.

3. Let $K$ denote an arbitrary total quotient ring. For a valuation ring $V$ of $K$ we have $V \subseteq T(V) \subseteq K$. It is easy to see that $V$ is also a valuation ring of $T(V)$. A ring $R$ is a Prüfer ring in case the rings between $R$ and $T(R)$ are integrally closed. In the classical case every valuation domain is a Prüfer domain. This is not true in rings with zero divisors, see [1] and [3]. The purpose of this section is to give some sufficient conditions on the ring $K$ that imply that every valuation ring of $K$ is Prüfer. These conditions are given in Lemma 3, Theorem 6, and Corollary 7.

A ring $R$ has few zero divisors if it has only finitely many maximal prime ideals of zero. $R$ is additively regular if, for each $z \in T(R)$, there is a $u \in R$ such that $z+u$ is a regular element of $T(R)$ (see [4]). The class of additively regular rings contains the set of rings with few zero divisors which, in 
turn, contains the set of integral domains and the set of Noetherian rings. A ring $R$ is $\pi$-regular in case, for each $r \in R$, there exists an $a \in R$ and a positive integer $n$ such that $r^{n}=\left(r^{n}\right)^{2} a$. Note that if $n=1$ we merely have the definition of a commutative von Neumann regular ring. Recall that a subdirect sum of rings $\left\{R_{\alpha}\right\}_{\alpha \in \mathscr{Q}}$ is a subring $S$ of the complete direct sum $\bigoplus \sum R_{\alpha}=\left\{f: \mathscr{A} \rightarrow \bigcup R_{\alpha} \mid f(\alpha) \in R_{\alpha}\right.$, for each $\left.\alpha \in \mathscr{A}\right\}$ with the property that for each $r_{\alpha} \in R_{\alpha}$, there exists $f \in S$ so that $f(\alpha)=r_{\alpha}$ [11]. We need the following two types of quotient rings. Let $N$ be a multiplicatively closed subset of $R$. Define

$$
\begin{aligned}
& R_{(N)}=\{a \mid b: a, b \in R, b \text { is a regular element of } N\} ; \\
& R_{[N]}=\{z \in T(R): z s \in R, \text { for some } s \in N\} .
\end{aligned}
$$

The ring $R_{[N]}$ is called the large quotient ring of $R$ with respect to $N$. If $P$ is a prime ideal of $R$ we write $R_{(P)}$ and $R_{[P]}$ in place of $R_{(R-P)}$ and $R_{[R-P]}$, respectively. A quasi-valuation ring is a ring $R$ with the property that for each regular element $x$ in $T(R), x$ or $x^{-1}$ is in $R$.

LEMMA 3. Let $R$ be an additively regular ring.

(a) $R$ is a valuation ring if and only if $R$ is a quasi-valuation ring.

(b) If $P$ is a prime ideal of $R$, then $R_{[P]}=R_{(P)}$.

(c) If $V$ is a valuation ring between $R$ and $T(R)$, then $V$ is a Prüfer ring.

Proof. (a) and (b) are in [4], and are easy generalizations of Lemmas 2 and 4 of [6].

(c) It is obvious that $V$ is additively regular. Let $M$ be a maximal ideal of $V$. Then (a) and (b) imply that $V_{[M]}=V_{(M)}$ is a valuation ring. By Theorem 13 of [6], $V$ is a Prüfer ring.

LEMMA 4. (a) If $K$ is a $\pi$-regular ring and if $V$ is a valuation ring of $K$; then $T(V)$ is a $\pi$-regular ring, or $V=T(V)$.

(b) If $K$ is a von Neumann regular ring and if $V$ is a valuation ring of $K$; then $T(V)$ is a von Neumann regular ring, or $V=T(V)$.

Proof. Recalling that $\left(\mathrm{V}_{2}\right)$ is equivalent to $\left(\mathrm{V}_{4}\right)$, we let $P$ (resp., $v$ ) be the prime (resp., valuation) associated with the valuation ring $V$ as in $\left(\mathrm{V}_{4}\right)$ (resp., $\left(\mathrm{V}_{2}\right)$ ). The ideal $P_{\infty}=\{x \in T(V): v(x)=\infty\}$ is a prime ideal in both $V$ and $T(V)$.

(a) If $V$ contains no regular nonunits, then $V$ is a total quotient ring and is therefore equal to $T(V)$. So we assume the existence of at least one regular nonunit $b$ of $V$. We must show that if $z \in T(V)$, then there is an $a \in T(V)$ and a positive integer $n$ such that $z^{2 n} a=z^{n}$.

Case 1. If $z \notin V$, then there is an $a \in K$ such that $z^{2 n} a=z^{n}$ for some $n$. But, $n v(z)=-v(a)<0$ implies that $a \in V \subseteq T(V)$. 
Case 2. Assume that $z \in V-P$. Then $v(z / b)<0$. So by Case $1,(z / b)^{2 n} a=$ $(z / b)^{n}$, and thus $z^{2 n}\left(a / b^{n}\right)=z^{n}$ where $a / b^{n} \in T(V)$.

Case 3. Let $z \in P-P_{\infty}$. Since $V$ is a valuation ring of $T(V)$, we can find $y \in T(V)-V$ such that $z y \in V-P$. Say that $y=c / d$, where $d$ is a regular element of $R$. It follows that $z / d \notin P$. Use Case 1 if $z / d \in T(V)-V$, and use Case 2 if $z / d \in V-P$.

Case 4. Let $z \in P_{\infty}$, then there exist $a$ and $n$ as in Case 1 so that $z^{2 n} a=$ $z^{n}$. Also, $z^{2 n}\left(a^{2} z^{n}\right)=z^{n}$, and clearly $a^{2} z^{n} \in P_{\infty} \subseteq T(V)$. The proof of (b) follows from the proof of (a) by letting $n=1$.

Proposition 5. If $R$ is a ring whose total quotient ring $T(R)$ is $\pi$ regular, then $R$ is additively regular. In particular, every ring with von Neumann regular total quoteint ring is additively regular.

Proof. We first prove the result when $T(R)$ is assumed to be a von Neumann regular ring. It is well known that a von Neumann regular ring is a subdirect sum of a set of fields-say that $T(R)$ is a subdirect sum of the family of fields $\left\{F_{\alpha}\right\}_{\alpha \in \mathscr{A}}$. If $z \in T(R)$, write $z=a / b$ where $a, b \in R$ and $b$ is regular. Considering $a$ as an element of $T(R)$, there is an element $c \in T(R)$ such that $a^{2} c=a$. Write $c=c_{0} / c_{1}$ where $c_{i} \in R$ and $c_{1}$ is regular.

Define $u=\left(c_{1}-a c_{0}\right) b$ and let $t=z+u$. Our goal is to prove that $t$ is regular. This is equivalent to proving $t(\alpha) \neq 0$, for all $\alpha \in \mathscr{A}$. (Here we are treating $T(R)$ as a subdirect sum of $\left\{F_{\alpha}\right\}_{\alpha \in \mathscr{A}}$.) Fix $\alpha \in \mathscr{A}$, then $t(\alpha)=$ $a(\alpha) / b(\alpha)+\left[c_{1}(\alpha)-a(\alpha) c_{0}(\alpha)\right] b(\alpha)$. If $a(\alpha)=0$, then $t(\alpha)=c_{1}(\alpha) b(\alpha) \neq 0$. On the other hand, assume that $a(\alpha) \neq 0$. Then $c_{1}(\alpha)-a(\alpha) c_{0}(\alpha)=0$; for $a^{2} c=a$ implies that $a(\alpha)[a(\alpha) c(\alpha)-1]=0$, and hence

$$
a(\alpha) c(\alpha)-1=a(\alpha)\left[c_{0}(\alpha) / c_{1}(\alpha)\right]-1=0 .
$$

Thus $t(\alpha)=a(\alpha) / b(\alpha) \neq 0$.

Now assume that $T(R)$ is $\pi$-regular. Let $\left\{P_{\delta}\right\}$ be the set of prime ideals of $T(R)$ and let $N=\bigcap P_{\delta}$ be the nilradical of $N$. By relation (2), $R /(R \cap N) \subseteq$ $T(R) / N \subseteq T(R /(R \cap N))$ and since $T(R) / N$ is a von Neumann regular ring [12, Proposition 1], we must have $T(R) / N=T(R /(R \cap N))$. By the first part of the proof, $R /(R \cap N)$ is additively regular. Thus, if the coset $z+N$ is in $T(R) / N$, there exists a $u \in R$ such that $(z+N)+(u+N)=b+N$ is regular in $T(R) / N$. Clearly $z+u=b+n$, where $n \in N$. The regular element $b+N \notin P_{\delta} / N$ for all $\delta$, and thus $b \notin P_{\delta}$ for all $\delta$. It follows that $b+n$ is a regular element of $T(R)$. This completes the proof.

THEOREM 6. If $K$ is a $\pi$-regular ring, then every valuation ring of $K$ is a Prüfer ring.

Proof. Let $V$ be a valuation ring of $K$. By Lemma $4, V=T(V)$ or 
$T(V)$ is $\pi$-regular. In the first case $V$ is obviously Prüfer. In the second case invoke Lemma 3 and Proposition 5.

Returning to the situation in $\S 2$, we have

COROLlaRY 7. If $R$ is a reduced ring, then every valuation ring of $Q(R)$ is a Prüfer ring.

4. In this last section we consider rings with the following property:

(I) $R$ is integrally closed in $T(R)$ if and only if $R$ is the intersection of valuation rings between $R$ and $T(R)$.

If $R$ is a domain, then (I) is Krull's theorem. There are two results in the literature of a ring $R$ satisfying (I), when $R$ is assumed to have zero divisors. Griffin [6] and Larsen [9] proved independently that (I) holds for rings with few zero divisors. Also appearing in [6] is the result that if $T(R)$ is a von Neumann regular ring, then (I) holds for $R$. It is quite easy to see, using Griffin's methods, that additively regular rings satisfy (I). We give another class of rings satisfying (I).

PROPOSITION 8. Let $R$ be a ring such that $Q(R)$ is a von Neumann regular ring and such that every unit of $Q(R)$ is also a unit of $T(R)$. Then $R$ satisfies property (I).

Proof. Assume that $R$ is integrally closed in $T(R)$ and that $R^{\prime}$ is the integral closure of $R$ in $Q(R)$. By Proposition 2, $R^{\prime}=\bigcap V_{\alpha}$, where each $V_{\alpha}$ is a valuation ring of $Q(R)$. From relation (1) $R=\bigcap V_{\alpha}^{\prime}$, where $V_{\alpha}^{\prime}=$ $V_{\alpha} \cap T(R)$. To complete the proof we need show that each $V_{\alpha}^{\prime}$ is a valuation ring. To this end, we prove that each $V_{\alpha}^{\prime}$ is additively regular (for it is already a quasi-valuation ring). Let $z \in T(R)=T\left(V_{\alpha}^{\prime}\right)$. By Lemma 4 and Proposition $5, V_{\alpha}$ is additively regular. Choose $u \in V_{\alpha}$ so that $z+u=r$ is regular in $T\left(V_{\alpha}\right)$. Thus, $r$ is regular in $Q(R)$, so $r \in T(R)$, and hence $r-z=$ $u \in V_{\alpha}^{\prime}$. Therefore $V_{\alpha}^{\prime}$ is additively regular.

Rings satisfying the hypothesis of Proposition 8 are easy to construct. For example, for each natural number $i$, let $F_{i}$ be the prime field of characteristic 2, let $K$ be the complete direct sum of $\left\{F_{i}\right\}_{i=1}^{\infty}$, and let $R$ be the subring of $K$ generated by the discrete direct sum of $\left\{F_{i}\right\}_{i=1}^{\infty}$ and the identity element of $K$. Then $R=T(R), K=Q(R)$, and $Q(R) \neq T(R)$ since $(1,0,1,0, \cdots) \notin T(R)$. Clearly, every unit of $Q(R)$ is a unit of $T(R)$.

\section{BIBLIOGRAPHY}

1. M. Biosen and M. Larsen, Prüfer and valuation rings with zero divisors, Pacific J. Math. 40 (1972), 7-12.

2. N. Bourbaki, Eléments de mathématique, Fasc. 30. Algèbre commutative, Chap. VI, Actualités Sci. Indust., no. 1308, Hermann, Paris, 1964. MR 33 \#2660.

3. R. Gilmer, On Prüfer rings, Bull. Amer. Math. Soc. 78 (1972), 223-224. 
4. R. Gilmer and J. Huckaba, $\Delta$-rings, J. Algebra (to appear).

5. M. Griffin, Generalizing valuations to commutative rings, Queen's Math. Preprint No. 1970-40, Queen's University, Kingston, Ontario, 1970.

6. —_, Prüfer rings with zero divisors, J. Reine Angew. Math. 239/240 (1967), 55-67. MR 41 \#188.

7. W. Krull, Allgemeine Bewertungstheorie, J. Reine Angew. Math. 167 (1932), 160196.

8. J. Lambek, Lectures on rings and modules, Blaisdell, Waltham, Mass., 1966. MR 34 \#5857.

9. M. Larsen, Harrison primes in a ring with few zero divisors, Proc. Amer. Math. Soc. 22 (1969), 111-116. MR 40 \#1388.

10. M. Manis, Valuations on commutative rings, Proc. Amer. Math. Soc. 20 (1969), 193-198. MR 38 \#2134.

11. N. McCoy, The theory of rings, Macmillan, New York, 1964. MR 32 \#5680.

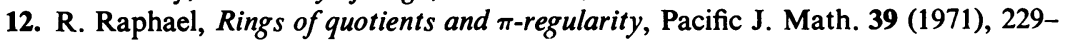
233.

13. P. Samuel, La notion de place dans un anneau, Bull. Soc. Math. France 85 (1957), 123-133. MR 20 \#7018.

Department of Mathematics, University of Missouri, Columbia, Missouri 65201 